\title{
Malignant epithelioid angiomyolipoma invading the inferior vena cava: Using a temporary vena cava filter to prevent tumour emboli during nephrectomy
}

\author{
Xiang Li, MD; Runming Liu, MD; Dalin He, MD \\ Department of Urology, First Affiliated hospital, Medical College, Xi'an Jiaotong University, Xi'an, Shaanxi, China
}

Cite as: Can Urol Assoc J 2014;8(7-8):e564-6. http://dx.doi.org/10.5489/cuaj.1814

Published online August 11, 2014.

\section{Abstract}

Angiomyolipoma (AML) is generally considered to be benign and malignant angiomyolipoma is rare. This paper presents an extremely rare case of epithelioid AML with tumour thrombus invading inferior vena cava (IVC). We present the case of a 36-year-old woman with epithelioid AML with tumour thrombus invading inferior vena cava who underwent radical nephrectomy and IVC thrombectomy. As an adjunctive procedure, a temporary IVC filter was placed in suprarenal position before operation. One week after surgery, the temporary IVC filter was retrieved by femoral approach. Three months postoperatively, a computed tomography scan and abdominal ultrasonogaphy showed no evidence of thrombus in IVC or renal vein and no sign of tumour recurrence. Epithelioid AML is extremely rare and can be malignant, with invasion of the IVC or renal vein. Implanting temporary filter can prevent fatal pulmonary complication and avoid potential the side effects of permanent filter.

\section{Introduction}

Renal angiomyolipoma (AML) is generally considered benign. However, very few cases indicate potential malignant behaviours, including regional lymph node involvement and renal vein or inferior vena cava (IVC) invasion. ${ }^{1-2}$ AML with malignant character are extremely rare. These patients need urgent surgical intervention for potential fatal pulmonary embolus complications.

For common renal cell carcinoma with tumour invading the IVC or renal vein, it is recommended to implant a suprarenal IVC filter before surgical treatment to reduce the risk of pulmonary embolus complications. A permanent IVC filter may result in serious complications, including renal vein or IVC thrombosis, extremity edema and even renal failure. ${ }^{3-4}$ However, the novel temporary IVC filter has mini- mal morbidity ${ }^{5}$ and is easy to insert and retrieve, without potential long-term complications. To our knowledge, this paper is the first to describe the use of a temporary IVC filter to prevent embolus complications for malignant $\mathrm{AML}$ with tumour thrombus invading the IVC.

\section{Case report}

A 36-year-old woman presented with right flank pain lasting 3 months. Her physical examination revealed a right renal mass and routine laboratory test results were unremarkable. The computed tomography (CT) scan showed a mass with low CT value in the right renal lower pole. Magnetic resonance images (MRI) showed adipose tissue area with negative intensity in the lesion, a high-intensity tumour in the right kidney, and a tumour thrombus in the main renal vein and inferior vena cava (Fig. 1).

The MRI and CT showed a tumour with fatty element in the right renal lower pole and the tumour thrombus extending into the IVC.

Based on preoperative examinations, this patient underwent radical nephrectomy and IVC thrombectomy using a midline transperitoneal approach. Before surgery, the diagnostically venacavography identified the level of tumour thrombus extension. Therefore, a temporary IVC filter (Cook, USA) was placed in the suprarenal position. To effectively clamp the IVC above the tumour, we ensured there was enough space between the filter and the tumour embolus.

After the patient had the filter for 4 days, we used a midline transperitoneal approach to fully mobilize the right kidney (Fig. 2). The tumour embolus and filter were identified in the renal vein and IVC. The next step was to control the IVC and left renal vein by using the Satinsky clamp. The tumour embolus was then safely removed by incision in the anterior cava wall. Following this procedure, the cavatomy was repaired and then the radical nephrectomy was successfully performed. No enlarged lymph nodes were observed. 


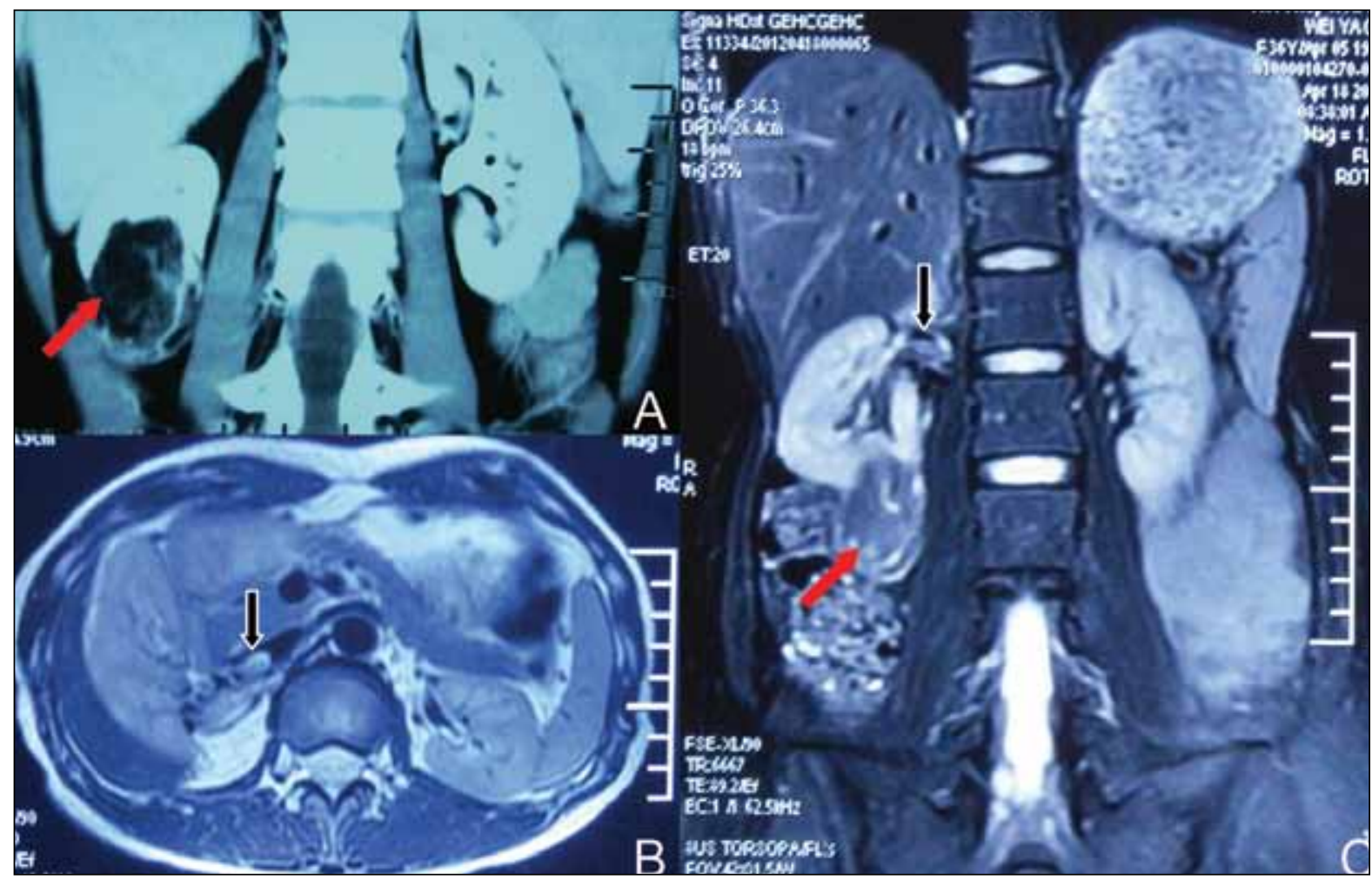

Fig. 1. Magnetic resonance images and computed tomography scan results.

One week after the surgery, the temporary IVC filter was removed using jugular approach.

Macroscopic examinations showed a $5 \times 5 \times 4-\mathrm{cm}$ tumour in the lower pole of the right kidney and a $3-\mathrm{cm}$ thrombus in renal vein and IVC. According to pathological analysis, the right renal mass was composed predominantly of mature fatty tissues, with smooth muscle cells and epithelioid cells around the blood vessels. The tumour cells were cytologically benign, without atypical or cellular nuclear mitosis (Fig. 3). Adjacent renal tissues and adrenal glands were normal.

\section{Discussion}

Renal angiomyolipoma is mesenchymal tumour with or without tuberous sclerosis. ${ }^{6}$ This tumour, mainly made up of adipose tissues, dystrophic vessels and smooth muscle cells, is generally benign. Angiomyolipoma with malignant characters is extremely rare.

Epithelioid AML is a subtype of angiomyolipoma. It is regarded as a potentially malignant tumour. This uncommon AML is composed of epithelioid cells around the vessels. Epithelioid AML generally occurs in patients with tuberous sclerosis. It may have a benign or rapidly progressive malignant course. It is not easy to predict whether the tumour is malignant or benign, even by histological examination. ${ }^{7}$ Epithelioid AML with tumours invading the renal vein or IVC carries the risk of potentially fatal cardiopulmonary embolism. Therefore, surgical treatments, including nephrectomy and tumour thrombectomy, are necessary even though patients are asymptomatic.

To decrease the risk of pulmonary embolism for those patients with tumour embolus, some surgeons recommend implanting the IVC filter before the nephrectomy. ${ }^{8-9}$ However, placing the filter is considered to increase the risk of thrombosis and inflammation. Generally, the permanent IVC filter may cause several complications, including extremity edema, infection and even organ's dysfunction. As for the temporary IVC filter, it minimized the risk of those complications and it is easy to insert and retrieve.

For patients without filter protection, the potential embolus detachment may cause fatal pulmonary embolus complications. During the process of mobilizing the kidney, surgeons should be very careful to minimize squeezing of the tumour tissues, to reduce the possibility of embolus shedding. Thus, we prefer to recommend placing the filter before surgery to avoid those fatal complications.

In this case report, we present a 36-year-old woman with right epithelioid $\mathrm{AML}$, who was otherwise healthy. The tumour invaded the right renal vein and IVC. She underwent radical nephrectomy and tumour thrombectomy. To avoid fatal pulmonary embolism, we implanted a temporary suprarenal IVC filter. One week after surgery, the filter was retrieved using the jugular approach. Three months postop- 


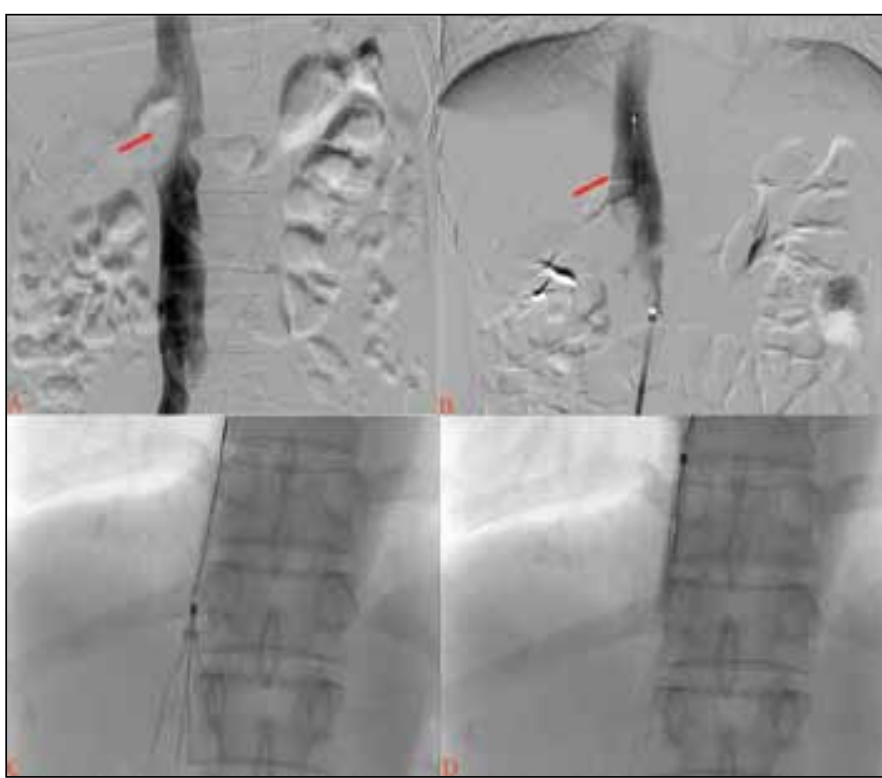

Fig. 2. The inferior vena cava (IVC) venography and implanting or retrieval of the temporary filter. A: The red arrow shows the filling defect with length of 3 $\mathrm{cm}$ in the IVC, which generally indicates an embolus into the vein chamber. B: A specially designed filter (red arrow) was placed above the embolus using the femoral approach. C and D: Specially designed retrieval equipment was used to close the filter by the jugular vein approach and to remove the filter.

eratively, a CT scan and abdominal ultrasonogaphy showed no evidence of thrombus in the IVC or renal vein, and no tumour recurrence.

\section{Conclusion}

Epithelioid $A M L$ is very rare. It has malignant potential and can invade the IVC or renal vein. Implanting a temporary IVC filter can prevent fatal pulmonary complications and reduce the risk of potential side effects of a permanent IVC filter. Further clinical investigation is still needed to establish its utility in a larger number of patients.

Competing interests: Dr. Li, Dr. Liu and Dr. He all declare no competing financial or personal interests.

This paper has been peer-reviewed.

\section{References}

1. Mittal V, Aulakh BS, Daga G. Benign renal angiomyolipoma with inferior vena cava thrombosis. Urology 2011;77:1503-6. http://dx.doi.org/10.1016/i.urology.2011.01.039

2. Faraji H, Nguyen BN, Mai KT. Renal epithelioid angiomyolipoma: A study of six cases and a metaanalytic study. Development of criteria for screening the entity with prognostic significance. Histopathology 2009;55:525-34. http://dx.doi.org/10.1111/i.1365-2559.2009.03420.x

3. Habito CR, Kalva SP. Inferior vena cava filter thrombosis: A review of current concepts, evidence, and approach to management. Hosp Pract 2011;39:79-86. http://dx.doi.org/10.3810/hp.2011.08.583

4. Kuo WT, Tong RT, Hwang GL, et al. High-risk retrieval of adherent and chronically implanted IVC filters: Techniques for removal and management of thrombotic complications. J Vasc Interv Radiol 2011;20:154856. http://dx.doi.org/10.1016/i.jvir.2009.08.024

5. Rosenthal $D$, Wellons ED, Levitt $A B$, et al. Role of prophylactic temporary inferior vena cava filters placed at the ICU bedside under intravascular ultrasound guidance in patients with multiple trauma. J Vasc Surg 2004;40:958-64. http://dx.doi.org/10.1016/i.jvs.2004.07.048

6. Sato K, Ueda Y, Tachibana H, et al. Malignant epithelioid angiomyolipoma of the kidney in a patient with tuberous sclerosis: An autopsy case report with p53 gene mutation analysis. Pathol Res Pract 2008;204:771-7. http://dx.doi.org/10.1016/i.prp.2008.04.008

7. Li J, Zhu M, Wang Y. Malignant epithelioid angiomyolipoma of the kidney with pulmonary metastases and p53 gene mutation. World J Surg Oncol 2012;8:213. http://dx.doi.org/10.1186/1477-7819-10-213

8. Wellons E, Rosenthal D, Schoborg T. Renal cell carcinoma invading the inferior vena cava: use of a "temporary" vena cava filter to prevent tumor emboli during nephrectomy. Urology 2004;63:380-2. http://dx.doi.org/10.1016/j.urology.2003.10.010

9. Carrafiello $G$, Mangini $M$, Fontana $F$, et al. Suprarenal inferior vena cava filter implantation. Radiol Med 2012;117:11. http://dx.doi.org/10.1007/s11547-012-0851-5

Correspondence: Dr. Dalin He, No.277 Yanta West Road, Xi'an, Shaanxi Province, China; Urologist1x@163.com

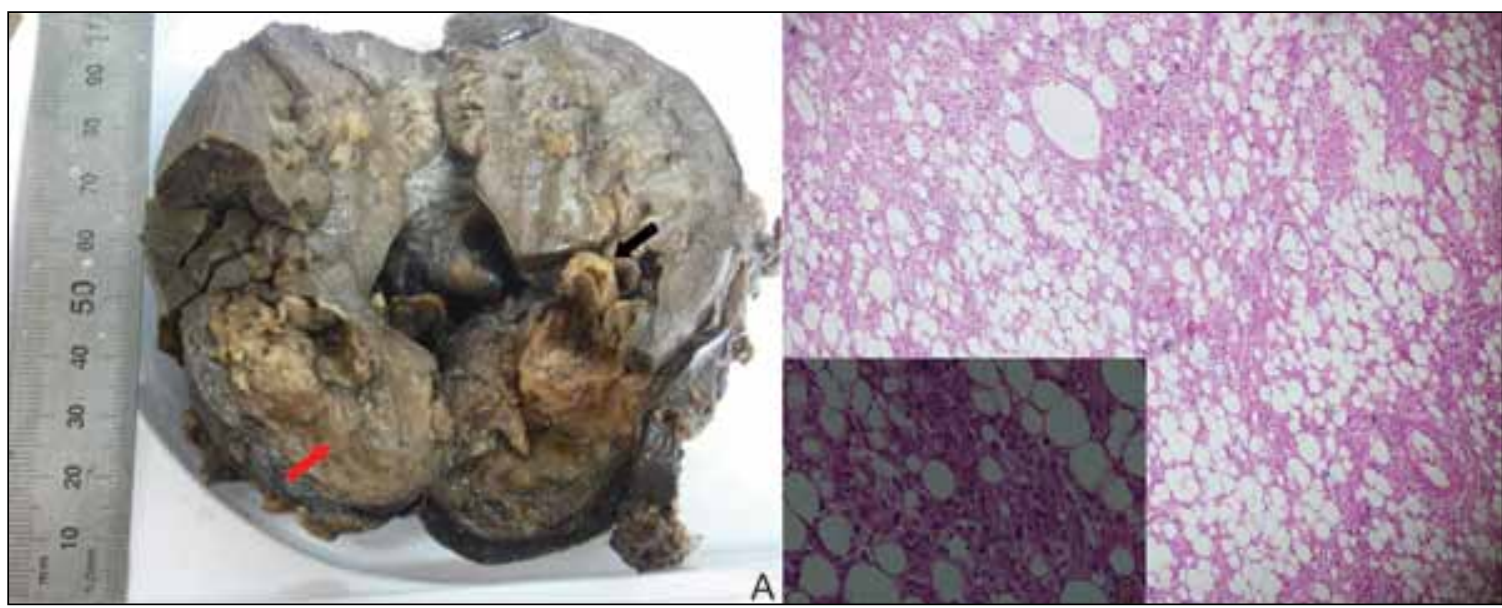

Fig. 3. Macroscopic and pathological findings. Macroscopic examination showed a tumour (red arrow) with size of $5 \times 5 \times 4 \mathrm{~cm}$ in the lower pole of the kidney. The part of the embolus was also observed in the renal vein (black arrow). Microscopic examination revealed numerous epithelioid cells around the tumour components, including fatty tissues, blood vessels and smooth muscle tissues. Cytomorphology analysis indicated that most of these epithelioid cells were polygonal in shape and variable in size. 